\title{
Deformation Calculating of Electromagnetic Launcher's Rail Subjected to Logarithmic Magnetic Pressure
}

\author{
Wen Liu (Corresponding author) \& Min Li \\ School of Sciences, Yanshan University, Qinhuangdao 066004, China \\ Xiangzhong Bai \\ School of Civil Engineering \& Mechanics, Yanshan University, Qinhuangdao 066004, China \\ E-mail: liuwen1961@hotmail.com \\ Leilei Wang \\ School of Sciences, Yanshan University, Qinhuangdao 066004, China
}

This project is supported by National Natural Science Foundation of China (Grant NO. 50875230).

The Excellent Going Abroad Experts' Training Program in Hebei Province.

\begin{abstract}
To solve the accurate calculation of force-deformation of the electromagnetic launcher's rail, this is helpful to extend the rail life and improve the firing accuracy. Therefore, the electromagnetic launcher's rail can be modeled as a beam on elastic foundation with simply supported beam by moving load. In this paper Euler beam theory is applied to build the Mechanical model and the analytical solution of the equation subjected to logarithmic magnetic pressure is derived in detail, which has successfully avoided the errors which are caused by using the uniform pressure to approximately replace the variable force. The numerical analysis brings from the elastic coefficient, the damping coefficient, the mass of rail and the load's velocity have influence on the deformation of beam by the MATLAB software. The consequence shows that the elastic coefficient and the load's velocity have quite obvious affect on the deformation of the beam while the damping coefficient and the mass of rail have not obvious affect on the deformation of the beam. It laid the foundation for solve the electromagnetic launcher's rail subjected to magnetic pressure of arbitrary function and promote the practicality of the electromagnetic guns.
\end{abstract}

Keywords: Electromagnetic gun, Launcher's rail, Elastic foundation beam, Damping force, Mechanical model, Lagrange equation

\section{Introduction}

The electromagnetic gun is a new concept weapon, the technology of its has inestimable application potential not only in the military field, but also in aviation, aerospace, transportation, industrial production, scientific research and other fields. Since the 80 s, especially in the recent ten years, with the development of new technology and new material, the research of launcher, launching weight, projectile velocity and high efficiency power source in the electromagnetic railgun have reached a series of achievement. The Su Rense.Livermore Nation Laboratory and The Lowes.Alamos Nation Laboratory, once have cooperated to accelerated a projectile weighed $2.2 \mathrm{~g}$ to a supervelocity of $10 \mathrm{~km} / \mathrm{s}$. Fluid Physics Institute of the Chinese Engineering Academy had built the first electromagnetic rail launcher, which can accelerate the projectile weighted $0.34 \mathrm{~g}$ to $16.8 \mathrm{~km} / \mathrm{s}$. While the velocity of the conventional cannon is only $2 \mathrm{~km} / \mathrm{s}$, which is so closed to the limitation of physics that the range is not possible to be farther. On the contrary, the thrust of the electromagnetic railgun is ten times bigger than that of the traditional launcher. The projectile can be accelerated to several kilometers or even to dozens of kilometers in one second, for it possesses huge kinetic energy which greatly enhance the range and power of the weapon.

T.Tzeng used the elastic foundation beam to build mechanical model of the electromagnetic railgun and deduced the solve process of governing equation. HU Yuwei analyzed theoretical built model and simulation analysis for the work of the process of electromagnetic railgun. WANG Sheng adopted the Fourier transform to study displacement field due to a moving load on Euler beam resting on an elastic half-space.

However, the damping force to the response of beam is ignored in the above researches. There is no doubt that 
the calculation of this kind of situation has the defects of analysis and calculation of mechanical. As a high-tech and high-precision electromagnetic launcher, accurate theoretical analysis and calculation in engineering are require. But until now, no researcher has given any exact analytical solution, thus further analytic solution of the equation subjected to variable pressure is of great significance. In fact, it is of theoretical value to research the theoretical analytic solution of various disciplines, including the analytical solution of practical engineering problems. On the one hand, its mechanical picture can be completely stated, on the other hand it can be used as a standard solution, to widely produce variety of numerical solution.

In this paper, regarding the rail as simply supported beam on the elastic foundation and considering the damping force, a mechanic model which is under the effect of moving load is proposed . Moreover, making use of variable method and the Lagrange equation which considering the damping force, the analytical solution (Liu Wen and San Rui,2009)of the governing equation subjected to nonlinear function pressures is derived and the influences brought from the elastic coefficient, the damping coefficient, the mass of rail and the load's velocity on the response of beam is analyzed.

\section{Mechanical Model}

Fig.1 shows a schematic of an electromagnetic railgun composed of power source, rail, armature and projectile. When the electric current of armature goes through the rail, it forms a strong magnetic field in the area of their encirclement. With the reaction by the magnetic field and the electric current, it emerges powerful electromagnetic force, which pushes the armature and projectile to do the accelerating motion along the rail till the projectile be launched out of the rail.

Fig. 2 is the mechanical model of the railgun - simply supported beam partially subjects to nonlinear load in a time-varying region sitting on the elastic foundation. Considering the effect of the beam by the damping force and basing on the Euler beam theory, we obtain the governing equation of elastic foundation beam by moving load which is a transient fourth-order differential equation as follows (S.Timoshenko,1965 and YU Yanli,2002):

$$
m \frac{\partial^{2} w}{\partial t^{2}}+E I \frac{\partial^{4} w}{\partial x^{4}}+k w+c \frac{\partial w}{\partial t}=p(x, t)
$$

Where $w$ is the deflection, $m=\rho B h$ is the mass per unit length, $\rho$ is the density of rail material, $B$ and $h$ are respectively the width and thickness of the rail, $E I$ is the bending stiffness of beam, $k$ is the elastic constant, $c$ is the damping coefficient. The function $p(x, t)=q(x)[1-H(x-v t)] \operatorname{in}(1)$, represents the magnetic pressure front traveling along the rail with velocity $v$ represented by a Heaviside step function $H(x-v t)$ ( Jerome T. Tzeng,2005), and $q(x)=q \log _{a}(x) \quad, a>1$.

\section{Solution of the Homogeneous Equation}

The homogeneous equation is a fourth—order partial differential equation, in order to change it into the ordinary differential equation, we solve it by the method of variable separation.

The solution of the homogeneous equation of (1)can be expressed as follows:

Substituting(2)into the homogeneous equation of(1):

$$
w(x, t)=\phi(t) \theta(x)
$$

$$
m \frac{\partial^{2} \phi}{\partial t^{2}} \theta+E I \phi \theta^{(4)}+k \phi \theta+c \theta \frac{\partial \phi}{\partial t}=0
$$

That can be expressed as follows:

from equation(4), let:

$$
-\frac{\frac{\partial^{2} \phi}{\partial t^{2}}}{c \phi}-\frac{\partial \phi}{m \phi}=\frac{E I}{c m} \frac{\theta^{(4)}}{\theta}+\frac{k}{c m}
$$

$$
-\frac{\frac{\partial^{2} \phi}{\partial t^{2}}}{c \phi}-\frac{\frac{\partial \phi}{\partial t}}{m \phi}=\lambda^{2}
$$

And 


$$
\frac{E I}{c m} \frac{\theta^{(4)}}{\theta}+\frac{k}{c m}=\lambda^{2}
$$

That is

$$
\theta^{(4)}-\beta^{4} \theta=0
$$

Where, $\beta^{4}=\left(\lambda^{2}-\frac{k}{c m}\right) \frac{c m}{E I}$

solution of equation(5)can be expressed as follows:

$$
\phi(t)=A e^{\frac{-c+R}{2 m} t}+B e^{\frac{-c-R}{2 m} t}
$$

Where, $R^{2}=c^{2}-4 c m^{2} \lambda^{2}>0$

Based on the boundary condition of the simple beam,

$$
\left\{\begin{array} { c } 
{ \theta ( 0 ) = 0 } \\
{ \frac { \partial ^ { 2 } \theta ( x ) } { \partial x ^ { 2 } } | _ { x = 0 } = 0 }
\end{array} \quad \text { and } \quad \left\{\begin{array}{c}
\theta(L)=0 \\
\left.\frac{\partial^{2} \theta(x)}{\partial x^{2}}\right|_{x=L}=0
\end{array}\right.\right.
$$

solution of equation(7)can be expressed as follows( ZHU Shijian,2006):

$$
\theta_{i}(x)=\sqrt{\frac{2}{m L}} \sin \frac{n \pi}{L} x
$$

Accordingly:

$$
w_{i}(x, t)=\theta_{i}(x) \phi_{i}(t)=\left(\sqrt{\frac{2}{m L}} \sin \frac{n \pi}{L} x\right) \times\left[A_{i} e^{\frac{-c+R}{2 m} t}+B_{i} e^{\frac{-c-R}{2 m} t}\right]
$$

In terms of the orthogonality of $\theta_{i}(x)$ (ZHANG Xiangting,2006), we obtain:

$$
\int_{0}^{L} \theta_{i} \theta_{j} d x= \begin{cases}0, & \text { at } i \neq j \\ 1, & \text { at } i=j\end{cases}
$$

Hence, deformation $w(x, t)$ of the beam can be expressed by the linear combination of $\theta_{i}(x)$.

$$
w(x, t)=\sum_{i} \theta_{i}(x) \phi_{i}(t)=\sum_{i}\left(\sqrt{\frac{2}{m L}} \sin \frac{n \pi}{L} x\right) \times\left[A_{i} e^{\frac{-c+R}{2 m} t}+B_{i} e^{\frac{-c-R}{2 m} t}\right]
$$

Where constants $A_{i}, B_{i}$ are determined by the initial conditions.

\section{Analytical Solution of Governing Equation}

The analytical solution of (1) can be obtained by the Lagrange equation including the damping force. Where $T$ is the kinetic energy of the beam, $U$ is the total stain energy, $G$ is the dissipation function(ZHANG Xiangting,2002).

$$
\frac{d}{d t}\left(\frac{\partial T}{\frac{\partial \phi_{i}}{\partial t}}\right)-\frac{\partial T}{\partial \phi_{i}}+\frac{\partial U}{\partial \phi_{i}}-\frac{\partial G}{\frac{\partial \phi_{i}}{\partial t}}=Q_{i}
$$

The kinetic energy of the beam $T$ can be expressed as follows (LOU Ping,2003):

$$
T=\frac{1}{2} \sum_{i} M_{i}\left(\frac{\partial \phi_{i}}{\partial t}\right)^{2}
$$

Where $M_{i}=\int_{0}^{L} m \theta_{i}^{2}(x) d x$ represents the general mass of the beam.

The total strain energy of the beam $U$ is consisted by the strain energy $U_{b}$ of the beam, and the strain energy $U_{f}$ of 
the foundation.

$$
\begin{gathered}
U_{b}=\frac{1}{2} \int_{0}^{L} E I\left(\frac{\partial^{2} w(x, t)}{\partial x^{2}}\right)^{2} d x=\frac{1}{2} \sum_{i} \sum_{j} \phi_{i} \phi_{j} \int_{0}^{L} E I \frac{\partial^{2} \theta_{i}}{\partial x^{2}} \frac{\partial^{2} \theta_{j}}{\partial x^{2}} d x=\frac{1}{2} \frac{E I}{m} \sum_{i} \beta_{i}^{4} M_{i} \phi_{i}^{2} \\
U_{f}=\frac{1}{2} \int_{0}^{L} k w^{2} d x=\frac{k}{2} \int_{0}^{L}\left(\sum_{i} \theta_{i} \varphi_{i}\right)^{2} d x=\frac{k}{2} \sum_{i} \sum_{j} \phi_{i} \phi_{j} \int_{0}^{L} \theta_{i} \theta_{j} d x=\frac{k}{2 m} M_{i} \sum_{i} \phi_{i}^{2}
\end{gathered}
$$

The total strain energy $U$ is obtained as:

$$
U=U_{b}+U_{f}=\frac{1}{2} \frac{E I}{m} \sum_{i} \beta_{i}^{4} M_{i} \phi_{i}^{2}+\frac{k}{2 m} M_{i} \sum_{i} \phi_{i}^{2}=\frac{1}{2}\left[\sum_{i} M_{i} \phi_{i}^{2}\left(\frac{E I}{m} \beta_{i}^{4}+\frac{k}{m}\right)\right]=\frac{1}{2}\left[\sum_{i} c M_{i} \phi_{i}^{2} \lambda_{i}^{2}\right]
$$

The dissipation function $G$ can be expressed as:

$$
G=-\frac{1}{2} \int_{0}^{L} c\left(\frac{\partial w}{\partial t}\right)^{2} d x=-\frac{1}{2} \sum_{i} \sum_{j} \frac{\partial \phi_{i}}{\partial t} \frac{\partial \phi_{j}}{\partial t} \int_{0}^{L} c \theta_{i} \theta_{j} d x=-\frac{c}{2 m} \sum_{i} M_{i}\left(\frac{\partial \phi_{i}}{\partial t}\right)^{2}
$$

The virtual work done by the magnetic pressure $p(x, t)=q(x)[1-H(x-v t)]$ in a virtual displacement $\delta \varphi_{i}$ can be expressed as follows:

$$
\partial W=\int_{0}^{L} p(x, t) \delta w_{i} d x=\sum_{i} \partial \phi_{i} \int_{0}^{L} p(x, t) \theta_{i}(x) d x=\sum_{i} \partial \phi_{i} Q_{i}
$$

Where we define $Q_{i}$ as the generalized force

$$
\begin{aligned}
Q_{i} & =\int_{0}^{L} p(x, t) \theta_{i}(x) d x=\int_{0}^{v t} q(x) \theta_{i}(x) d x=\sqrt{\frac{2}{m L}} \int_{0}^{v t} q \log _{a}(x) \sin \frac{n \pi x}{L} d x \\
& =\frac{q \sqrt{\frac{L}{2 m}}}{n \pi \ln (a)}\left[2 \ln (v t)\left(1-\cos \left(\frac{n \pi v t}{L}\right)\right)+C_{1} e^{t}\right]
\end{aligned}
$$

Substituting $T, U, G, Q_{i}$ into the Lagrange equation including the damping force, we obtain an ordinary differential equation:

$$
\frac{\partial^{2} \phi_{i}}{\partial t^{2}}+\frac{c}{m} \frac{\partial \phi}{\partial t}+c \lambda_{i}^{2} \phi_{i}=\frac{Q_{i}(t)}{M_{i}}=F(t)
$$

Where:

$$
\begin{aligned}
F(t) & =\int_{0}^{L} p(x, t) \theta_{i}(x) d x=\int_{0}^{v t} q(x) \theta_{i}(x) d x=\sqrt{\frac{2}{m L}} \int_{0}^{v t} q \log _{a}(x) \sin \frac{n \pi x}{L} d x \\
& =\frac{q \sqrt{\frac{L}{2 m}}}{M_{i} n \pi \ln (a)}\left[2 \ln (v t)\left(1-\cos \left(\frac{n \pi v t}{L}\right)\right)+C_{1} e^{t}\right]
\end{aligned}
$$

The general solution of equation(9)is:

$$
\begin{aligned}
\phi_{i}(t) & =\frac{m}{R}\left(\frac{\partial \phi_{i}(0)}{\partial t}+\frac{c+R}{2 m} \phi_{i}(0)\right) e^{\frac{-c+R_{t}}{2 m}}+\frac{m}{R}\left(-\frac{\partial \phi_{i}(0)}{\partial t}+\frac{-c+R}{2 m} \phi_{i}(0)\right) e^{\frac{-c-R_{t}}{2 m}} \\
& +\frac{m}{R} \int_{0}^{t} F(\xi)\left(e^{\frac{-c+R}{2 m}(t-\xi)}-e^{\frac{-c-R^{2}}{2 m}(t-\xi)}\right) d \xi
\end{aligned}
$$

So the general solution of(1)can be expressed as follows:

$$
\begin{aligned}
w(x, t)= & \sum_{i} \theta_{i}(x) \phi_{i}(t)=\sum_{i} \sqrt{\frac{2}{m L}} \sin \frac{n \pi}{L} x\left\{\frac{m}{R}\left(\frac{\partial \phi_{i}(0)}{\partial t}+\frac{c+R}{2 m} \phi_{i}(0)\right) e^{\frac{-c+R}{2 m} t}\right. \\
& +\frac{m}{R}\left(-\frac{\partial \phi_{i}(0)}{\partial t}+\frac{-c+R}{2 m} \phi_{i}(0)\right) e^{\frac{-c-R}{2 m} t} \\
& \left.+\frac{m}{R} \int_{0}^{t} F(\xi)\left(e^{\frac{-c+R}{2 m}(t-\xi)}-e^{\frac{-c-R}{2 m}(t-\xi)}\right) d \xi\right\}
\end{aligned}
$$

The initial conditions are as:

$$
\left\{\begin{array}{c}
\phi(0)=0 \\
\left.\frac{\partial \phi(t)}{\partial t}\right|_{t=0}=0
\end{array}\right.
$$

So 


$$
\phi_{i}(t)=\frac{m}{R} \int_{0}^{v \xi} F(\xi)\left(e^{\frac{-c+R}{2 m}(t-\xi)}-e^{\frac{-c-R}{2 m}(t-\xi)}\right) d \xi
$$

According, substituting the solution of (11) into the equation (10), so we can get the solution $w(x, t)$ of (1). The moment and the shear force of the beam in the rail can be further derived from $w(x, t)$, which provides basis for the overall investigation of the dynamic behavior of the electromagnetic railgun.

\section{Numerical analysis}

Since there are differences among materials of electromagnetic rail launcher, the damping force and the rate of moving load will possibly bring influence to the response of the rail(Jerome T. Tzeng 2005, 41: 246-250.). Thus, it is necessary to consider the elastic coefficient, the damping coefficient, the mass of rail and the load's velocity to compare the response of the rail.

A known Material is modulus of rail material $E=120 \mathrm{GPa}$, the elastic constant $k=2.532 \times 10^{10} \mathrm{~N} / \mathrm{m}^{2}$, the density of rail material $\rho=8700 \mathrm{~kg} / \mathrm{m}^{3}$, the width of rail $B=3 \times 10^{-2} \mathrm{~m}$, the thickness of rail $h=1 \times 10^{-2} \mathrm{~m}$, the length of rail $L=2 \mathrm{~m}$, the magnetic load collection degree $\mathrm{q}(x)=110 \sin x \mathrm{MPa}(\mathrm{JF}, 2006)$.

Fig. 3 shows the deformation of the beam by the elastic coefficient. Along with the elastic coefficient $(k)$ increasing, the curve of time-deformation is a decreasing trend. Under the calculating conditions given by this paper, for the rail of which $k$ equals to $2.532 \times 10^{10} \mathrm{~N} / \mathrm{m}^{2}$, the deformation $(w)$ of the beam is $-0.9 \times 10^{-3} \mathrm{~m}$, when the armature moves to the moment $t=1 \times 10^{-3}$. While for the rail of which $k$ equals to $5.064 \times 10^{10} \mathrm{~N} / \mathrm{m}^{3}$, the deformation $(w)$ of the beam is $-0.2 \times 10^{-3} \mathrm{~m}$ at the same moment, we can see that the former is $78 \%$ smaller than the latter.

Fig. 4 shows the deformation of the beam by the damping coefficient. Along with the damping coefficient $(c)$ increasing, the curve of time-deformation is a slowly decreasing trend.

Fig. 5 shows the deformation of the beam by the mass of rail. Compared with copper and aluminum rail, the curve of time-deformation has no significant changes.

Fig.6 shows the deformation of the beam by the load's velocity. Along with the load's velocity $(v)$ increasing, the curve of time-deformation is a increasing trend. Under the calculating conditions given by this paper, for the rail of which $v$ equals to $1000 \mathrm{~m} / \mathrm{s}$, the deformation $(w)$ of the beam is $1.7 \times 10^{-3} \mathrm{~m}$ when the armature moves to the moment $t=1.6 \times 10^{-3} \mathrm{~s}$. While for the rail of which $v$ equals to $1200 \mathrm{~m} / \mathrm{s}$, the deformation $(w)$ of the beam is $3.1 \times 10^{-3} \mathrm{~m}$ at the same moment, we can see that the former is $82 \%$ smaller than the latter.

\section{Conclusions}

(1)Taking the rail as a simply support beam on the elastic foundation and considering the damping force, a mechanical model for the electromagnetic railgun is built.

"We don't have general solution to nonlinear problems and some particular solutions are as few as treasures in history."( Zheng Zhemin,1994) In this paper, making use of variable method and the Lagrange equation including the damping force, the general solution of the homogeneous part and the analytical solution of the governing equation subjected to logarithmic pressures is derived which enriched and developed the theory of elastic mechanics with the hope to lay the foundation for solving the difficulty problem of electromagnetic rail subjected to arbitrary distribution function pressures.

(2)The deformation of beam which is influenced by the elastic coefficient, the damping coefficient, the mass of rail and the load's velocity are analyzed by the MATLAB software. When the elastic coefficient is larger, the deformation of beam is smaller; when the load's velocity is larger, the deformation of beam is larger, the damping coefficient and the mass of rail have not obvious affect on the deformation of the beam.

\section{References}

Anthony J. Johnson1 and Francis C.Moon2. (2006). Elastic Waves and Solid Armature Contact Pressure in Electromagnetic Launchers Transactions on Magnetics 2006.3, 472-475.

HU Yuwei. (2007). Modeling and simulation of electromagnetic rail gun system[D]: [Dissertation for the Master Degree]. Harbin Institute of Technology, 2007.

HU Yuwei. (2007). Modeling and simulation of electromagnetic railgun system[D]: [Dissertation for the Master Degree]. Harbin Institute of Technology, 2007.

Jerome T. Tzeng and Wei Sun. (2007). Dynamic Response of Cantilevered Rail Guns Attributed to Projectile/Gun Interaction -Theory. Transactions on Magnetics, 2007, 43: 207-213. 
Jerome T. Tzeng. (2005). Dynamic Response of Electromagntic Railgun Due to Projectile Movement Transactions on Magnetics, 2005, 41: 246-250.

Jerome T. Tzeng. (2005). Structural Mechanics for Electromagnetic Railguns Transactions on Magnetics, 2005,1, 246-250.

JIN Shangnian, MA Yongli. (2002). Theoretical Mechanics. Higher Education Press, 2002, 211-226.

Liu Wen, San Rui. Mathematic Model and Analytic Solution for Cylinder Subject to Exponential Function. Chinese Journal of Mechanical Engineering, 2009,22(4) : 587-593.

Liu Wen. Mathematic Model and Analytic Solution for Cylinder Subject to Uneven Pressures. Chinese Journal of Mechanical Engineering , 2006,19(4) 574-578.

LOU Ping,ZENG Qingyuan. (2003). Finite element analysis of infinitely long beam resting on continuous viscoelastic foundation subjected to moving loads. Journal of Traffic and Tran sportation Engineering 2003, 3: $1-6$.

S.Timoshenko. (1965). Mechanics of material. Science press 1965,1-15.

Wang sheng. (2007). Displacement field due to a moving load on Euler beam resting on an elastic half-space [D]: [Dissertation for the Master Degree]. Harbin Institute of Technology, 2007.

WANG Ying, XIAO Feng. (1994). Principle of electricgun. National Defense Industry Press, 1994. 5-16.

YU Yanli. (2002). The research of the dynamic response of the rail system and viaduct by moving load [D]: [Dissertation for the Master Degree]. Wuhan University of Technology, 2002.

ZHANG Xiangting, WANG Zhipei. (2006). Structure Vibration Mechanics. TongJi Univesity Press 2006, 93-117.

Zheng Zhemin. ZhengZheMin corpus. (1994). Beijing: science press, 1994. P286http://www.sj00.com/soft/1481.htm

ZHU Shijian, LOU lingjun. (2006). Vibration Theory and Vibration Isolation. National Defense Industry Press 2006,36-66.

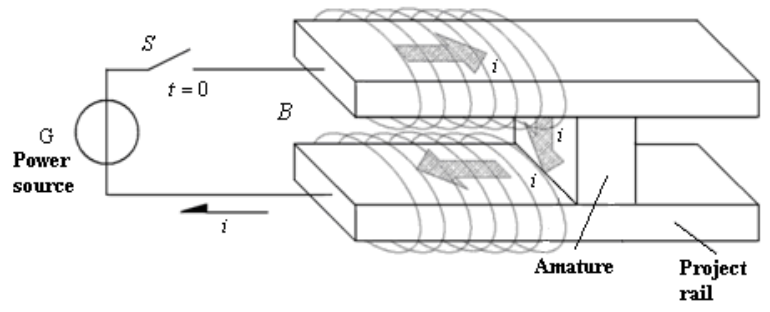

Figure 1. The general diagram of the railgun

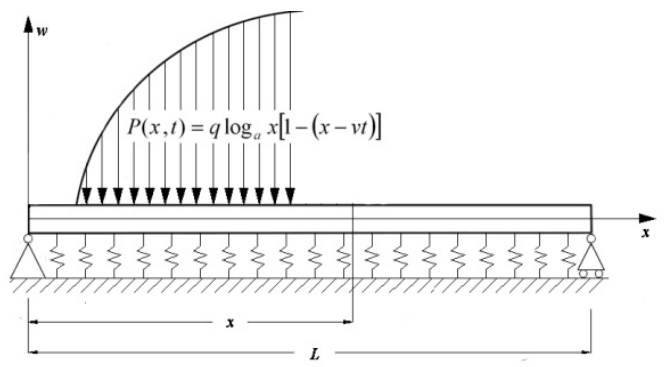

Figure 2. The rail is modeled as a beam on elastic foundation 


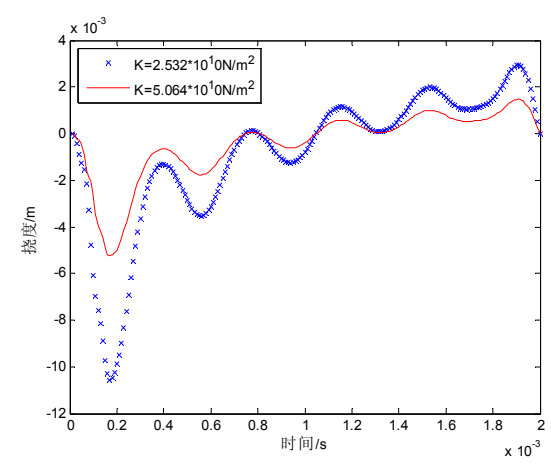

Figure 3. Deformation curve by differences elastic coefficient

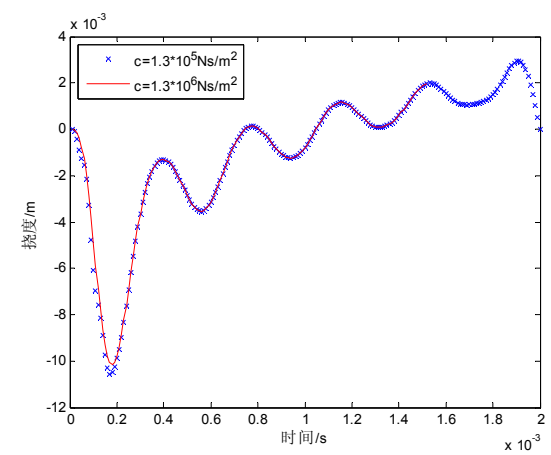

Figure 4. Deformation curve by differences damping coefficient

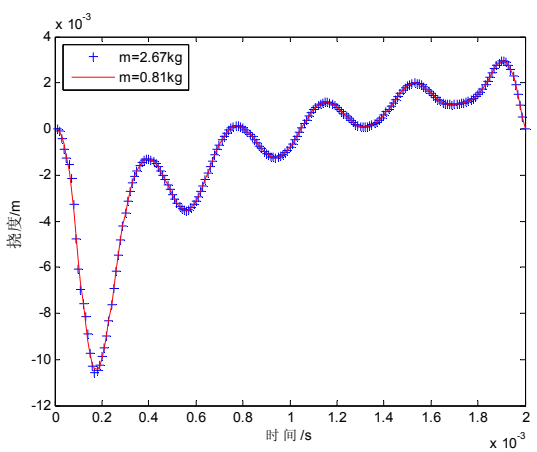

Figure 5. Deformation curve by differences mass of rail

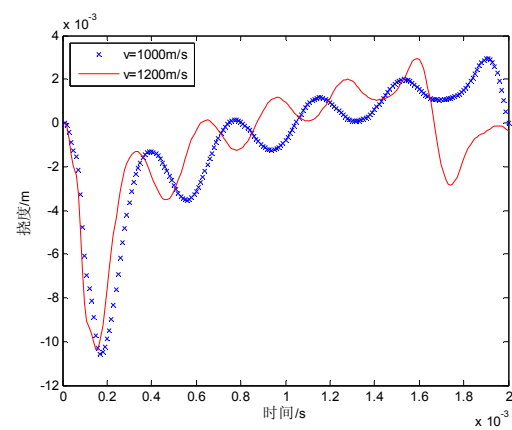

Figure 6. Deformation curve by differences velocity of the load 